\title{
FORMA PULMONAR CRÔNICA DA ESQUISTOSSOMOSE MANSONI. AVALIAÇÃO CLÍNICO-RADIOLÓGICA
}

\author{
Regina Lunardi Rocha, Enio Roberto Pietra Pedroso, \\ Manoel Otávio da Costa Rocha, José Roberto Lambertucci, \\ Dirceu Bartolomeu Greco e Cid Sérgio Ferreira
}

\begin{abstract}
Foram estudados 115 pacientes esquistossomóticos, 31 com radiologia torácica normal sem sinais de hipertensão pulmonar (HP); $73 \mathrm{com}$ alterações radiológicas cardiopulmonares sem sinais de HP e 11 com alteraçóes clínicas de HP. A forma pulmonar crônica (FPC) sem HP é de alta incidência e benigna. Nao se associa à forma hepatosplênica (FHE) da esquistossomose mansoni, à faixa etária, sexo ou naturalidade. As alterações radiológicas torácicas predominantes são hilares, seguidas das parenquimatosas (micronodulação, especialmente base direita). Associa-se às cargas parasitárias baixa ou média. A FPC com HP é de baixa incidência, mas determina repercussão cardiaca significativa. Associa-se à faixa etária superior a 12 anos e a FHE; não se relaciona ao sexo, cor e naturalidade. As alterações radiológicas torácicas são observadas no hilo e parênquima em igual proporção (arco médio abaulado e micronodulação em ambas as bases).

Palavras-chaves: Hipertensão pulmonar esquistossomótica. Esquistossomose pulmonar. Radiologia pulmonar esquistossomótica.
\end{abstract}

O comprometimento pulmonar na esquistossomose mansoni crônica ainda é pouco estudado. É encontrado, dependendo da casuística, em 3,8 a $86 \%$ dos pacientes esquistossomóticos 2102730 .

A forma pulmonar crônica (FPC) pode ocorrer sem hipertensão pulmonar (HP), com HP e cor pulmonale com ou sem cianose e HP discreta ou ausente e sem cor pulmonale 262728 .

A forma cardiopulmonar incide preferencialmente na faixa etária entre 10 e 30 anos de idade 5931 . Está quase sempre associada à forma hepatesplênica (FHE) especialmente à fibrose Symmers - Boglio$l^{3} 112430$.

As lesōes vasculares pulmonares podem ocorrer em conseqüência à: a) ação direta dos ovos e vermes (reação hiperérgica), 30 b) hipersensibilidade, sem relação direta com presença de ovos, $1013 \mathrm{c}$ ) hipertensão pulmonar ${ }^{7}$. As repercussões hemodinâmicas na fase crônica da esquistossomose pulmonar estão diretamente relacionadas com a extensão e gravidade das lesōes articulares pulmonares 123111219 .

As alterações funcionais pulmonares são decorrentes de insuficiência: a) ventilatória pulmonar; b)

\footnotetext{
Núcleo de Estudos sobre Esquistossomose Mansoni, Faculdade de Medicina da Universidade Federal de Minas Gerais. Financiado parcialmente pelo CNPq.

Endereço para correspondência: Dr. Enio Roberto Pietra Pedroso. Departamento de Clinica Médica/FM/UFMG. Av. Alfredo Balena 190 - s/439, Santa Efigênia, 30130 Belo Horizonte, MG, Brasil.

Recebido para publicação em 07/12/88.
}

alvéolo-respiratória; ou c) alvéolo-respiratória associada à insuficiência ventilatória discreta ou intensa. Não há correlação entre provas funcionais pulmonares e quadros clínico e radiológico cardiopulmonares.

$O$ objetivo deste trabalho é estudar em esquistossomóticos as alterações clínico-radiológicas cardiopulmonares em função da HP, correlacionado o tipo de alterações radiológicas cardiopulmonares (CP), com formas anátomo-clínicas, idade, sexo, cor, naturalidade, eletrocardiografia (ECG), eosinófilos no escarro e sangue, a função pulmonar e carga parasitária.

\section{MATERIAL E MÉTODOS}

Foram estudados 115 pacientes esquistossomóticos (Tabela 1), divididos em três grupos: I) 31 pacientes com radiologia do tórax normal, sem sinais clínicos sugestivos de hipertensão porta (grupo controle); II) 73 pacientes com alterações radiológicas cardiopulmonares, sem sinais clínicos de HP (forma pulmonar sem HP); III) 11 pacientes com alterações clínicas de HP (forma pulmonar com HP), subdividido em IIIA - sem alterações radiológicas cardiopulmonares (quatro pacientes) e IIIB - com alterações radiológicas cardiopulmonares (sete pacientes); Tabela 1.

Avaliação clínica: 1) critério diagnóstico da forma intestinal (FI): presença de ovos viáveis de $S$. mansoni nas fezes e exclusão de outras parasitoses e outras formas de esquistossomose; 2) critério diagnóstico da FHE: presença de hepatesplenomegalia ao exame clínico, confirmada à laparoscopia, presença de varizes do esôfago ao estudo radiológico, esplenopor- 
Rocha RL, Pedroso ERP, Rocha MOC, Lambertucci JR, Greco DB, Ferreira CS. Forma pulmonar crôniça da esquistossomose mansoni. Avaliação clínico-radiológica. Revista da Sociedade Brasileira de Medicina Tropical 23:83-89, abr-jun, 1990

Tabela 1 - Distribuição dos pacientes com esquistossomose mansoni em função da faixa etária, cor, sexo, formas anátomoclínicas.

\begin{tabular}{|c|c|c|c|c|c|c|c|c|c|c|c|c|c|c|}
\hline \multirow[t]{2}{*}{$\begin{array}{l}\text { Alteração } \\
\text { Pulmonar }\end{array}$} & \multicolumn{3}{|c|}{ Faixa Etária } & \multicolumn{5}{|c|}{ Cor } & \multicolumn{3}{|c|}{ Sexo } & \multicolumn{3}{|c|}{$\begin{array}{c}\text { Formas } \\
\text { Anátomo-Clínicas }\end{array}$} \\
\hline & $4 \vdash 12$ & $12 \vdash 20$ & $20 \vdash 44$ & $\mathbf{T}$ & $\mathrm{L}$ & $\mathrm{Fa}$ & Me & $\mathrm{T}$ & M & F & $\mathrm{T}$ & I & HE & $\mathrm{T}$ \\
\hline Ausente & 13 & 15 & 3 & 31 & 16 & 10 & 5 & 31 & 23 & 8 & 30 & 23 & 8 & 30 \\
\hline Presente sem HP & 34 & 39 & 2 & 73 & 45 & 16 & 12 & 73 & 44 & 29 & 73 & 50 & 23 & 73 \\
\hline Presente com HP & 1 & 9 & 1 & 11 & 5 & 5 & 1 & 11 & 6 & 5 & 11 & 2 & 9 & 11 \\
\hline Total & 58 & 53 & 6 & 115 & 66 & 31 & 18 & 115 & 73 & 42 & 115 & 75 & 40 & 115 \\
\hline
\end{tabular}

HP: hipertensão pulmonar, L: leucodermo, Fa: faiodermo, Me: melanodermo, M: masculino, F: feminino, I: intestinal, HE: hepatesplênica, T: total.

tografia, biópsia hepática; 3) critério diagnóstico da hipertensão pulmonar: hiperfonese da segunda bulha (B2) em área pulmonar (AP), com o segundo componente mais intenso que o primeiro $(\mathrm{C} 2>\mathrm{C} 1)$, estalido protosistólico (EPS) e terceira bulha (B3) do ventrículo direito (VD), com sobrecarga ventricular direita (SVD) ao ECG, isolados ou em associação.

Foram excluidos pacientes com esquistossomose mansoni ${ }^{21}$ ou formas associadas (enterobacteriose septicêmica prolongada, hepatites viróticas), os com doenças concomitantes (parasitoses intestinais, calazar). Foram também excluídos pacientes com hábito de tabagismo. A tuberculose pulmonar e as pneumonias inespecificas foram excluidas com base nos dados epidemiológicos, clinicos, radiológicos, laboratoriais (Gram e Pesquisa de Baar no escarro, PPD).

Avaliação laboratorial: constou do hemograma, reticulócitos, exame do esfregaço de sangue periférico, leucócitos (global, diferencial), plaquetas, hemossedimentação, exame de urina (caracteres gerais, elementos anormais, sedimentoscopia), transaminases (TGO, TGP), fosfatase alcalina (FA), bilirrubinas (BD, BT), uréia, creatinina, glicemia de jejum, exame coprológico ${ }^{15}$ e/ou retosigmoidoscopia (com biópsia retal e oograma qualitativo), ECG, função pulmonar (vitalografh e peak flow), eosionófilos no escarro. A biópsia hepática e hemoculturas foram realizadas para exclusão de concausas infectuosas e outras hepatopatias. A carga parasitária foi considerada baixa menos de 100 ovos/g de fezes, média de 100 a 400 ovos/g e alta 400 ovos/g. 1725 A eosinofilia sangüinea foi considerada aumentada em valores acima de $450 / \mathrm{mm}^{3}$.

Avaliaçāo radiológica: baseou-se na telerradiografia do tórax em PA e perfil esquerdo. Foram utilizados os seguintes critérios para análise das radiografias: a) hilo direito: adultos, chapas bem inspiradas (diafragma no 9. espaço intercostal direito), com $13 \mathrm{~mm}$ de largura e variação de $1 \mathrm{a} 3 \mathrm{~mm}$; no homem até $16 \mathrm{~mm}$ e na mulher até $15 \mathrm{~mm}$ e, em chapas expiradas, $11 \mathrm{~mm}$ (no homem até $15 \mathrm{~mm}$ e na mulher até $14 \mathrm{~mm}$ ), em crianças o ramo principal até $6 \mathrm{~mm}$; b) hilo esquerdo: considerou-se haver alteração na morfologia radiológica da artéria pulmonar esquerda quando de sua simples visualização; c) nodulação: micronódulos (presença de hipotransparência de 1 a $3 \mathrm{~mm}$ de diâmetro), macronódulos (hipotransparência maior que $1 \mathrm{e}$ menor que três $\mathrm{cm}$ ), nódulos (tamanho intermediário entre micro e macronódulos). Foi anotada a localização dos nódulos nos campos pulmonares: hemitórax direito (HD) ou esquerdo (HE), posição superior, média ou inferior, lateral, central ou medial. Não foram considerados os nódulos calcificados, de aspecto residual; d) rosário: micronodulação em seqüência definida à semelhança de rosário, analisado também quanto à sua localização nos campos pulmonares; e) arco médio: côncavo (morfologia habitual), retificado e abaulado; f) silhueta cardíaca: normal ou aumentada, seguindo o critério de seu diâmetro transverso aumentado quando maior que a metade do diâmetro látero-lateral do tórax.

A leitura do exame radiológico foi realizada por três observadores, independentes, em simples-cego, e considerada alterada quando pelo menos duas impressões foram coincidentes.

Todos os pacientes ou seus responsáveis concordaram por escrito a se submeterem ao trabalho proposto.

Os dados foram comparados pelo teste do "quiquadrado" e o nivel mínimo aceitável de significância foi de $5 \%$.

\section{RESULTADOS}

Dentre os 115 pacientes estudados, 75 apresentavam FI e 40 FHE a FP estava presente em 52 $(69,4 \%)$ pacientes com FI, 50 sem $(96,2 \%)$ e dois sem HP (3,8\%). Nos pacientes com FHE, $32(80 \%)$ tinham FP, 23 sem $(72,8 \%)$ e nove com HP $(28,2 \%)$. Houve correlação significativa $(p<0,01)$ entre FHE e HP (Tabela 2). 
Rocha RL, Pedroso ERP, Rocha MOC, Lambertucci JR, Greco DB, Ferreira CS. Forma pulmonar crônica da esquistossomose mansoni. Avaliação clínico-radiológica. Revista da Sociedade Brasileira de Medicina Tropical 23:83-89, abr-jun, 1990

Tabela 2 - Distribuiçào dos pacientes com esquistossomose mansoni de acordo com as formas anátomo-clínicas em funcão da presenca da forma pulmonar com ou sem hipertensão pulmonar.

\begin{tabular}{|c|c|c|c|c|c|c|c|}
\hline \multirow{3}{*}{$\begin{array}{l}\text { Formas } \\
\text { Anátomo-clinicas }\end{array}$} & \multicolumn{7}{|c|}{ Forma Pulmonar } \\
\hline & \multicolumn{5}{|c|}{ Presente } & \multirow{2}{*}{$\begin{array}{l}\text { Ausente } \\
\text { (c) }\end{array}$} & \multirow[t]{2}{*}{ Total } \\
\hline & $\begin{array}{l}\text { Com HP } \\
\text { (a) }\end{array}$ & $(\%)$ & $\begin{array}{l}\text { Sem HP } \\
\text { (b) }\end{array}$ & $(\%)$ & $\mathbf{T}$ & & \\
\hline Intestinal & 2 & $(3,8)$ & 50 & $(96,2)$ & 52 & 23 & 75 \\
\hline Hepatesplênica & 9 & $(28,2)$ & 23 & $(72,8)$ & 32 & 8 & 40 \\
\hline Total & 11 & & 73 & & 84 & 31 & 115 \\
\hline
\end{tabular}

axb: $x^{2}=8,24(p<0,01)$; axc: $x^{2}=8,37(p<0,01) ; a+b x c: x^{2}=1,01 ;$ bxc: $x^{2}=0,12$. HP: hipertensão pulmonar, T: total.

Dentre os 115 pacientes, $80(69,6 \%)$ apresentavam alterações à telerradiografia do tórax, $91,2 \%$ sem e 8,8\% com HP. Dentre os com HP, $63,6 \%$ possuíam alterações radiológicas torácicas (sete em 11 pacientes) (Tabela 3 ).

Tabela 3 - Distribuiçào dos pacientes com esquistossomose mansoni estudados em função da presença de alteraçōes radiológicas pulmonares e hipertensāo pulmonar.

\begin{tabular}{lccc}
\hline \multirow{2}{*}{$\begin{array}{l}\text { Alterações Radiológicas } \\
\text { Pulmonares }\end{array}$} & \multicolumn{2}{c}{ Hipertensão Pulmonar } & \\
\cline { 2 - 3 } & Presente & Ausente & \\
\hline Presente & 7 & 73 & 80 \\
Ausente & 4 & 31 & 35 \\
\hline Total & 11 & 104 & 115 \\
\hline
\end{tabular}

$\mathrm{X}^{2}: 0,01$

Não houve diferença significativa nos grupos com e sem HP quanto a distribuição das lesões, agrupadas em hilares e/ou parenquimatosas (Tabela 4). Nos pacientes sem HP as alterações hilares
Tabela 4 -Alterações radiológicas pulmonares (hilares $e$ parenquimatosas) em função da hipertensão pulmonar em pacientes com esquistossomose mansoni.

\begin{tabular}{lccc}
\hline \multirow{2}{*}{$\begin{array}{l}\text { Alterações Radiológicas } \\
\text { Pulmonares }\end{array}$} & \multicolumn{2}{c}{ Hipertensão Pulmonar } & \\
\cline { 2 - 3 } & Presente & Ausente & \\
\hline Hilares & 4 & 65 & 69 \\
Parenquimatosas & 3 & 8 & 11 \\
\hline Total & 7 & 73 & 80 \\
\hline
\end{tabular}

$X^{2}: 3,12$

representavam $87,7 \%$ do acometimento enquanto as parenquimatosas contribuiram com $10,9 \%$. Já na FP com HP, as alterações hilares e parenquimatosas foram de 57,1 e $42,9 \%$, respectivamente. Em ambos os grupos, com e sem HP, dentre as lesões parenquimatosas, a micronodulação foi de $91,0 \%$. No grupo com HP a micronodulação acometeu sempre ambos os hemitoraces, enquanto no grupo sem HP houve predominância do acometimento na base direita (Tabela 5).

Tabela 5 - Alteraçôes radiológicas cardiopulmonares em pacientes com esquistossomose mansoni em função da hipertensāo pulmonar.

\begin{tabular}{|c|c|c|c|c|c|c|}
\hline & \multirow{3}{*}{ Alterações Radiológicas } & \multicolumn{4}{|c|}{ Hipertensão Pulmonar } & \multirow{3}{*}{ Total } \\
\hline & & \multicolumn{2}{|c|}{ Ausente } & \multicolumn{2}{|c|}{ Presente } & \\
\hline & & No & $(\%)$ & No & $(\%)$ & \\
\hline & Arco médio retificado & 32 & $(43,8)$ & 1 & $(14,2)$ & 33 \\
\hline & Arco médio abaulado & 32 & $(43,8)$ & 3 & $(42,9)$ & 35 \\
\hline & Micronodulação HTD' & 5 & $(6,9)$ & 3 & $(42,9)$ & 8 \\
\hline & Micronodulação HTD e E & 2 & $(2,7)$ & 0 & - & 2 \\
\hline & Rosário HTD e E & 1 & $(1,4)$ & 0 & - & 1 \\
\hline & Área cardíaca aumentada & 1 & $(1,4)$ & 0 & - & 1 \\
\hline Total & & 73 & $(100,0)$ & 7 & $(100,0)$ & 80 \\
\hline
\end{tabular}

HTD: hemitórax direito, E: esquerdo, no = número. 
Rocha RL, Pedroso ERP, Rocha MOC, Lambertucci JR, Greco DB, Ferreira CS. Forma pulmonar crônica da esquistossomose mansoni Avaliação clínico-radiológica. Revista da Sociedade Brasileira de Medicina Tropical 23:83-89, abr-jun, 1990

A HP foi observada em 11 dos 115 pacientes $(9,6 \%)$. Os achados clínicos principais foram hiperfonese de $\mathrm{B} 2 \mathrm{em} \mathrm{AP}$, desdobramento de $\mathrm{B} 2 \mathrm{em} \mathrm{AP}$, fixo, com $\mathrm{C} 2>\mathrm{C} 1$, presentes em nove $(81,8 \%)$ dos 11 pacientes, O EPS de VD foi observado em dois entre 11 pacientes $(18,2 \%)$ e B3 de VD em um $(9,1 \%)$.

Houve associação significativa entre a presença de HP e faixa etária acima de 12 anos de idade (Tabela 6) mas não houve correlação com sexo ou cor.

Tabela 6 - Hipertensão pulmonar em função da faixa etária em pacientes comforma pulmonar da esquistossomose mansoni.

\begin{tabular}{lccc}
\hline \multirow{2}{*}{ Faixa Etária } & \multicolumn{3}{c}{ Hipertensão Pulmonar } \\
\cline { 2 - 3 } & Ausente & Presente & \\
\hline$<12$ & 34 & 1 & 35 \\
$\geq 12$ & 39 & 10 & 49 \\
\hline Total & 73 & 11 & 84 \\
\hline
\end{tabular}

$X^{2}: 4,09(p<0,05)$

A carga parasitária média ou baixa associou-se com alterações radiológicas pulmonares em pacientes sem HP (Tabela 7).

Tabela 7 - Alterações radiológicas pulmonares em função da carga parasitária em pacientes com forma pulmonar crônica da esquistossomose mansoni sem hipertensão pulmonar.

\begin{tabular}{lccc}
\hline \multirow{2}{*}{ Carga Parasitária } & \multicolumn{2}{c}{ Alterações Radiológicas } \\
\cline { 2 - 3 } & Ausente & Presente & \\
\hline Alta & 11 & 15 & 26 \\
Média ou baixa & 8 & 39 & 47 \\
\hline Total & 19 & 54 & 73 \\
\hline
\end{tabular}

$\mathrm{X}^{2}: 4,39(\mathrm{p}<0,05)$

O estudo da função pulmonar das grandes vias aéreas não permitiu diferenciar os pacientes com FPC sem HP tenham eles alterações radiológicas pulmonares ou não.

Também não houve diferenças quanto a eosinofilia sangǘnea ou no escarro entre os pacientes com FP com ou sem HP.

A presença de varizes do esôfago se associou ao grupo com FP e HP $(\mathrm{p}<0,01)$; Tabela 8 .
Tabela 8 - Hipertensão pulmonar em função da presença de varizes do esôfago ao estudo radiológico em pacientes com esquistossomose mansoni crônica com alterações radiológicas pulmonares.

\begin{tabular}{lccc}
\hline Varizes & \multicolumn{2}{c}{ Hipertensão Pulmonar } & \\
\cline { 2 - 3 } Esofageanas & Ausentes & Presentes & \\
\hline Presente & 6 & 5 & 11 \\
Ausente & 58 & 1 & 59 \\
\hline Total & 64 & 6 & 70 \\
\hline $\mathrm{X}^{2}: 17,3(\mathrm{p}<0,01)$ & & &
\end{tabular}

\section{DISCUSSÃO}

A maioria das observações da presença de FP da esquistossomose mansoni procede de material obtido de necrópsia ou biópsia. Koppisch (1941) 16 encontrou lesões pulmonares em 4,3\% dos casos de infecção esquistossomática leve, $23 \%$ em infecção moderada e $63,6 \%$ nos graves. As formas cardiopulmonares surgem em $26,2 \%{ }^{9}$ dos casos de acometimento pulmonar. Faria 10 observou $8,8 \%$ de cor pulmonale que se eleva para $12,9 \%$ ao se considerar apenas em relação aos portadores de FHE. Santiago e Ratton 27 ao estudarem 54 pacientes com FHE associada ao comprometimento pulmonar, encontraram 24 casos com HP e cor pulmonale $(44,4 \%)$ e seis casos com cianose, HP e cor pulmonale $(11,1 \%)$.

Os pacientes aqui descritos apresentavam ovos de $S$. mansoni nas fezes, tinham alterações radiológicas cardiopulmonares semelhantes às descritas por outros autores em relação à esquistossomose pulmonar, 11 deles apresentavam sinais clínicos sugestivos de HP, e foram afastadas outras etiologias que não a esquistossomótica pelos exames complementares. $\mathrm{O}$ quadro descrito é altamente sugestivo de esquistossomose pulmonar mesmo se ressentindo da resposta terapêutica e/ou da biópsia pulmonar como critérios diagnósticos. 91419

Neste trabalho, utilizando-se critérios clíni$\cos ^{29}$ observou-se incidência de $9,6 \%$ de HP entre os 115 pacientes estudados, representando $13,1 \%$ dentre as formas pulmonares crônicas, $22,5 \%$ dentre portadores de FHE da doença e 13,7\% dentre os 80 que apresentavam alterações radiológicas torácicas. Apesar de se utilizar critérios clínicos os dados se aproximam dos observados em necrópsias, o que enfatiza a importância do exame clínico no diagnóstico do comprometimento pulmonar esquistossomótico concordando com a correlação observada por Barbato², valorizando o médico e seus sentidos naturais na avaliação dos pacientes esquistossomóticos. 
Rocha RL, Pedroso ERP, Rocha MOC, Lambertucci JR, Greco DB, Ferreira CS. Forma pulmonar crônica da esquistossomose mansoni. A valiação clínico-radiológica. Revista da Sociedade Brasileira de Medicina Tropical 23:83-89, abr-jun, 1990

As alterações radiológicas cardiopulmonares foram observadas em $69,6 \%$ dos 115 casos estudados; $63,5 \%$ deles sem HP e 6,1\% com HP2 918 . As alterações radiológicas torácicas foram observadas nos pacientes com FHE em 72,5\% (29 pacientes), percentuali muito superior ao encontrado por Elian e Coura 68 . Só a biópsia pulmonar poderá trazer a resposta sobre qual o dado verdadeiro, mas mesmo assim a análise anátomo-patológica pode não distinguir repercussões funcionais (decorrentes de substâncias vasopressoras) sobre os vasos pulmonares ainda sem correspondência anatômica (por exemplo abaulamento ou retificação do arco médio). Nos pacientes com FP sem HP, aqui estudados, o achado mais observado foi, ao estudo radiológico do tórax, espessamento hilar, seguido de acentuação da estriação normal e presença de artérias em rosário. Ao se instalar a HP surge retificação ou abaulamento do arco médio ou ainda dilatação das câmaras direitas. Os micronódulos, nesses casos, se tornam escassos. Nos pacientes aqui descritos o quadro radiológico concordou com o observado por todos os autores, houve nítida predominância do acometimento hilar, seguido de alterações parenquimatosas focais ou disseminadas e acometimento cardíaco inexpressivo. Só não houve coincidência quanto aos valores estatísticos. A diferença deveu-se especificamente ao percentual de acometimento hilar. Rocha 25 ao estudar as alterações clínicas e radiológicas encontrou $58 \%$ de acometimento hilar em 24 individuos sadios de seu grupo-controle. Estes indivíduos estavam na segunda década de vida, à semelhança da casuística aqui apresentada, na qual $41,7 \%$ dos pacientes tinham de quatro a 12 anos e $54,8 \%$ de 12 a 20 anos. Nesta faixa etária, as alterações do arco médio podem ser encontradas irdependentemente da presença de patologia inclusive esquistossomótica. Pelo menos alguma parte das alterações hilares aqui observadas pode constituir o padrão normal do indivíduo e não secundária à infecção esquistossomótica. Mesmo não havendo diferença significativa nos grupos com ou sem HP quanto a distribuição das lesões no hilo ou parênquima, ou tipo de alteração do arco médio, observa-se que nos pacientes com FP sem HP as alterações hilares representaram $87,7 \%$ do acometimento, enquanto as parenquimatosas significaram $10,9 \%$, ficando o restante $(1,4 \%)$ por conta de um paciente que apresentou aumento discreto da área cardíaca. Já nos pacientes com FP e HP as lesões hilares representaram $57,1 \%$ e as parenquimatosas $42,9 \%$ das alterações radiológicas torácicas. No grupo com HP a micronodulação ocorreu em ambos os HT e bases, nos sem HP houve predominio na base direita mas sem significância estatística. Santiago e Ratton $\mathbf{2 6}$ ja haviam observado predominância de alterações na base direita no grupo sem HP. Esses dados associam a esquistossomose pulmonar a fatores ligados a local preferencial de fluxo sangüíneo pulmonar (bases e preferencialmente à direita), relacionam as alterações especialmente ao acometimento vascular parenquimatoso e reforçam o fator mecânico como responsável pelo acometimento pulmonar à semelhança dos observados por Main$\operatorname{zer}^{18}$.

A HP se relacionou à faixa etária além dos 12 anos. A forma cardiopulmonar tem seu pico máximo de incidência entre 10 a 30 anos. $\mathrm{O}$ acometimento nesta faixa etária é explicável pelo tempo necessário para o desenvolvimento da HP, pela resisténcia aumentada do hospedeiro após os 30 anos de idade, além de sobrevivência improvável após os 40 anos nos portadores desta forma ${ }^{5}$. Essa associação demonstra a necessidade de periodo de latência para o surgimento de HP, o que é favorável ao hospedeiro uma vez que o acometimento pulmonar pode ser reconhecido e a doença tratada especificamente, antes da instalação da HP. Não se encontrou associação significativa entre FP e sexo, cor, naturalidade, como observados por Coura ${ }^{6}$. É bem reconhecido o comportamento dos negros frente à esquistossomose, predominando neles formas benignas. Dos 115 pacientes, 18 eram negros $(15,6 \%)$ e $98(84,4 \%)$ leucodérmicos ou faiodérmicos. Dos 18 melanodérmicos, um (5,5\%) apresentava $\mathrm{HP}$, enquanto no grupo de 98 pacientes constituídos por leucodérmicos ou faiodérmicos, 10 tinham HP (10,3\%). Fica a impressão de que realmente brancos e faiodermos tendem a desenvolver quadros de maior gravidade embora a comparação entre os dados não seja significativa. Houve associação de FP com HP e FHE. A presença de hipertensão portal favorece a fuga mesentéricopulmonar de modo que os ovos são drenados continuamente para a árvore arterial pulmonar. A associação entre FHE e FP reforça a teoria mecânica da HP. 131130

A HP surgiu em 11 pacientes representando $9,6 \%$ de todo grupo estudado e $13,1 \%$ se se considerar os grupos com FP. Os principais achados de HP foram: hiperfonese de $\mathrm{B} 2$ em AP, desdobramento em AP com C2 > C1, SVD ao ECG, EPS em AT. Os dados aqui apresentados concordam com os descritos na literatura 414 e devem ser procurados enfaticamente. Sua caracterização justifica maior atenção e risco de evolução para formas cardiopulmonares.

A FPC com ou sem HP não se relacionou à presença no escarro de eosinófilos. Esse achado negativo permite diferenciar quadros radiológicos pulmonares de pneumonite esquistossomótica da pneumonite pós-tratamento especifico na qual o encontro de eosinófilos é característico. 2223 A ausência de eosinófilos no escarro e o encontro de função 
Rocha RL, Pedroso ERP, Rocha MOC, Lambertucci JR, Greco DB, Ferreira CS. Forma pulmonar crônica da esquistossomose mansoni. Avaliação clínico-radiológica. Revista da Sociedade Brasileira de Medicina Tropical 23:83-89, abr-jun, 1990

pulmonar (dependente das grandes vias áereas) incapaz de diferenciar pacientes com ou sem HP, pressupõe menor influência de reações de sensibilização brônquica às substâncias broncoespasmódicas na determinação da evolução da FPC. A presença de varizes do esôfago associadas à $\mathrm{HP}$ reforça a relação entre fuga mesentérica e embolização pulmonar de ovos, vermes e produtos de seu metabolismo à patogenia da esquistossomose pulmonar. $203032 \mathrm{~A}$ carga parasitária, entretanto, tendeu a ser média ou baixa nos pacientes com FP sem HP comparados ao grupo sem FP. Era de se esperar que sendo o fator mecânico primordial na patologia pulmonar quão maior a carga parasitária maior a probabilidade dos ovos atingirem aos pulmões. Desta forma, apesar de todos os indicios apontando a importância de fatores mecânicos no desenvolvimento da FP outros devem estar envolvidos na patologia pulmonar.

\section{SUMMARY}

One hundred and fifteen patients with schistosomiasis mansoni were studied: 31 with a normal chest $x$-rays and no signs of pulmonary hypertension $(P H)$ and 84 with $x$-rays compatible with cardiopulmonary abnormalities ( 73 without symptoms and 11 with symptoms of $\mathrm{PH})$. The chronic pulmonary form (CPF) without pulmonary hypertension is frequent and benign. There was no association between the CPF and the hepatosplenic form (HEF), nor with age, sex or patient origin. Pulmonary hilum alterations were the most common $x$-ray findings, followed by parenchymatous abnormalities (micronodules). The CPF was associated with a low to medium worm burden. The incidence of $C P F$ with pulmonary hypertension is low but usually related to significant heart abnormalities. It is more common in patients over 12 years, with the HEF of the disease and has no correlation with sex, race or place of birth. Chest $x$-ray alterations are seen with similar frequency both in parenchymatous and hilar regions.

Key-words: Pulmonary hypertension and schistosomiasis. Pulmonary involvement Schistosomiasis.

\section{REFERÊNCIAS BIBLIOGRÁFICAS}

1. Andrade ZA, Andrade SG. Pathogenesis of Schistosomal pulmonary arteritis. The American Journal of Tropical Medicine and Hygiene 19:305-10, 1970.

2. Barbato ECD. Pneumopatia e cor pulmonale crônico esquistossomóticos. Arquivos Brasileiros de Cardiologia 6:195-205, 1953.

3. Bogliolo L. Patologia. Revista Brasileira de Malariologia e Doenças Tropicais 11:359-424, 1959.

4. Cavalcanti IL, Tompson G. História natural e diagnóstico diferencial da hipertensão arterial pulmonar esquis- tossomótica. Jornal Brasileiro de Medicina 8:335-338, 1964.

5. Cavalcanti IL, Tompson G, Souza N, Barbosa FS. Pulmonary hypertension in schistossomiasis. British Heart Journal 24:363-371, 1962.

6. Coura JR. Esquistossomose pulmonar, estudo clínico e experimental. Cultura Médica. Rio de Janeiro, 1979.

7. Doss H. Pulmonary circulation and respiratory function. E. \& Livingstone, London, 1956.

8. Elian AA. Contribuição da biópsia pulmonar ao estudo da forma assintomática da esquistossomose mansoni pulmonar. Tese de Doutorado, Universidade Federal de Minas Gerais. Belo Horizonte, 119p, 1966.

9. Erfan M, Erfan H, Mousa AM, Dueeb AA. Chronic pulmonary schistosomiasis; clinical and radiological study. Transactions of the Royal Society Tropical Medicine and Hygiene 42:477-85, 1949.

10. Faria JL. Histopatologia da endarterite pulmonar esquistossomótica ( $S$. mansoni). Tese de Livre-Docência, Universidade Federal de Minas Gerais, Belo Horizonte, 1952.

11. Faria JL. Cor pulmonale in Manson's schistosomiasis; Frequency in necropsy material; pulmonary vascular changes caused by schistosome ova. American Journal of Pathology 30:167-193, 1954.

12. Faria JL, Barbas JV, Fujioka T, Lion MF, Andrade e Silva U, Décourt LV. Pulmonary schistosomatic arteriovenous fistulas producing a new cyanotic syndrome in Manson's schistosomiasis. American Heart Journal 58:556-567, 1959.

13. Jaffe $\mathbf{R}$ Observaciones sobre lesiones pulmonares producidas por Schistosoma mansoni (su frecuencia y su importancia). Revista de Sanidad y Assistencia Social 9:1287-1298, 1944.

14. Jiashun Z, Fuyuan F, Shuzhu Y, Guanghua L, Weiquing C, Songci L. Chronic cor pulmonale due Schistosoma japonicum infection. Chinese Medical Journal 94:52934, 1981.

15. Katz N, Chaves A, Pellegrino J. A simple device for quantitative stool thick-smear technique in schistosomiasis mansoni. Revista do Instituto de Medicina Tropical de São Paulo 14:397-400, 1972.

16. Koppisch E. Estudos sobre la esquistosomiasis de Manson en Puerto Rico: VI Anatomia patológica observada entre la población puertorriqueña. Puerto Rico Journal of Public Health Tropical Medicine 16:456-500, 1941.

17. Lambertucci JR, Pedroso ERP, Greco DB, Rocha MOC. Estabilidade na excreção de ovos de $S$. mansoni nas fezes de crianças com esquistossomose mansoni crônica. Revista do Instituto de Medicina Tropical de São Paulo 25:67-72, 1983.

18. Mainzer F. Clinical aspects of pulmonary diseases induced by Schistosoma haematobium and mansoni. Journal of the Egyptian Medical Association 21:762$795,1938$.

19. Meira JA. Estudo clínico das formas pulmonares da esquistossomíase mansônica (Doença de Manson Pirajá da Silva). Arquivos de Cirurgia Clínica e Experimental 6:3-136, 1942.

20. Menezes $H$. Experimental embolization of the pulmonary artery branches of dogs by adults Schistosoma 
Rocha RL, Pedroso ERP, Rocha MOC, Lambertucci JR, Greco DB, Ferreira CS. Forma pulmonar crônica da esquistossomose mansoni. Avaliação clínico-radiológica. Revista da Sociedade Brasileira de Medicina Tropical 23:83-89, abr-jun, 1990

mansoni. Revista do Instituto de Medicina Tropical de São Paulo 7:82-86, 1965.

21. Neves J. Como evoluem os seus pacientes com forma aguda da esquistossomose? In: Prata A, Aboim E (ed) Simpósio sobre Esquistossomose, Salvador, 1969. p. 192-199, 1970.

22. Pedroso ERP. Contribuição ao estudo da esquistossomose mansoni humana e experimental; especial referência às alterações clínico-radiológicas do pulmão após tratamento especifico, pulmão modelo imunopatológico da doença esquistossomótica. Tese de Doutorado. Universidade Federal de Minas Gerais, Belo Horizonte, 1982.

23. Pedroso ERP, Lambertucci JR, Rocha MOC, Greco DB, Raso P, Ferreira CS, Lima DP. Contribuiçăo da presença do eosinófilo no escarro ao diagnóstico das alterações pulmonares pós-tratamento na esquistossomose mansoni humana crônica. Estudo duplo-cego. Revista da Sociedade Brasileira de Medicina Tropical 17:77-79, 1984.

24. Raso P. Pedroso ERP. Esquistossomose Mansônica. In: Lopes ER, Chapadeiro E, Raso P, Tafuri WL (eds) Bogliolo-Patologia. Rio de Janeiro. Editora Guanabara, 4! edição, p. 1065-1086, 1987.

25. Rocha MOC. Estudo das alterações clínicas e radiológicas pulmonares da fase inicial da esquistossomose mansoni humana. Tese de Doutorado, Universidade Federal de Minas Gerais, Belo Horizonte, 1983.

26. Santiago JM, Ratton JL. Contribuição ao estudo radiológico da esquistossomose pulmonar. Revista da Associação Médica de Minas Gerais 15:2-7, 1964.

27. Santiago JM, Ratton JL. Formas clínicas da esquistossomose pulmonar crônica. Subsidios para a sua classificação. Revista da Associação Médica de Minas Gerais 19:62-81, 1968.

28. Santiago JM, Ratton JL. Quadro Clínico. Formas pulmonares. In: Cunha AS (org) Esquistossomose mansoni. São Paulo, Editora da Universidade de São Paulo, p. 170-183, 1970.

29. Santiago JM, Ratton JL, Malleta CA. Semiologia da hipertensão pulmonar. Revista da Associação Médica de Minas Gerais 17:66-76, 1966.

30. Shaw AFB, Ghareeb AA. Pathogenesis of pulmonary schistosomiasis in Egypt with special reference to Ayerza's disease. Journal of Pathology and Bacteriology 46:401-424, 1938.

31. Silva RM, Chaves E. Cor pulmonale crônico esquistossomótico III. Estudo clínico-patológico de 6 casos. O Hospital 60:455-470, 1961

32. Warren KS. Experimental pulmonary schistosomiasis. Transactions of the Royal Society of Tropical Medicine and Hygiene 58:228-233, 1964. 